\title{
Author Correction: Two large deletions extending beyond either end of the RHD gene and their red cell phenotypes
}

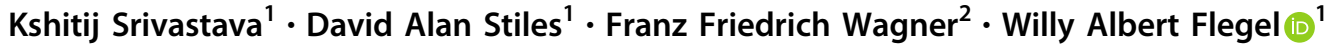

Published online: 1 May 2018

(c) The Author(s) under exclusive licence to The Japan Society of Human Genetics 2018

Correction to: Journal of Human Genetics advance online publication 16 November 2017; https://doi.org/10.1038/ s10038-017-0345-3

The authors of the above paper noticed an error in publication. In Results section, under sub-section RHD genetic variations, the deletion nomenclature for Sample 1 was incorrectly given as [NC_000001.11(NG_007494.1):c. (1-15149_1-15153)_(148+3154_148+3158)del].
The correct nomenclature for Sample 1 deletion is [NC_000001.11(NG_007494.1):c.(-15149_-15145)_(148+ 3154_148+3158)del].

This correction does not alter the results and their interpretation as discussed in the paper.

The authors would like to apologize for the error.

Willy Albert Flegel

waf@nih.gov

1 Department of Transfusion Medicine, Clinical Center, National Institutes of Health, MSC 1184, 10 Center Drive, Bethesda, MD 20892, USA

2 Red Cross Blood Service NSTOB, Institute Springe, Eldagsener Strasse 38, 31830 Springe, Germany 